\title{
Advanced method of scintillator energy resolution test
}

\author{
A.V.Gektin, V.S.Suzdal, A.Yu.Boyarintsev, A.V.Sobolev \\ Institute for Scintillation Materials, STC "Institute for Single Crystals", \\ National Academy of Sciences of Ukraine, \\ 60 Nauky Ave., 61072 Kharkiv, Ukraine
}

Received December 20, 2018

\begin{abstract}
This work is devoted to the development of advanced method of scintillator energy resolution test. Separation of scintillation photons for different groups in accordance with statistical methods allows to extract some groups of photons that give better resolution values comparing to conventional approach. It is shown that new method could be useful for analysis of scintillation uniformity of detector.
\end{abstract}

Keywords: scintillator, energy resolution, clusterization.

Разработан усовершенствованный метод измерения энергетического разрешения сцинтиллятора. Разделение сцинтилляционных фотонов на различные группы в соответствии со статистическими методами позволяет выделить группы фотонов, дающие лучшие значения разрешения по сравнению с традиционным подходом. Показано, что новый метод может быть полезен при анализе сцинтилляционной однородности детектора.

Вдосконалений метод вимірювання енергетичного розділення сцинтиляторів. О.В.Гектін,В.С.Суздаль, А.Ю.Бояринцев, О.В.Соболєв.

Розроблено вдосконалений метод вимірювання енергетичного розділення сцинтилятора. Розподілення сцинтиляційних фотонів на різні групи у відповідності до статистичних методів дозволяє виділити групи фотонів, які дозволяють отримати кращі значення енергетичного розділення у порівнянні із традиційним підходом. Показано, що новий метод може бути корисним при аналізі сцинтиляційної однорідності детектора.

\section{Introduction}

The history of the modern scintillation physics and engineering [1] gives us examples of the permanent studies and development. In [2] a description of the effects of statistics on resolution is provided. It based on simulation of theoretical limit for the light yield and energy resolution. It is shown that even the best parameters are far from the limit and this is the subject for the performance improvement.

In particular, a scintillator's resolution is most often quoted parameter for detector engineering. This is the reason for the permanent search of more efficient scin- tillators [3-5] and improvement of conventional scintillators $[6,7]$.

The energy resolution, $\Delta E / E$, of the full energy peak measured with a scintillator matched to a photomultiplier (PMT) can be written as [8, 9]:

$$
(\Delta E / E)^{2}=\left(\delta_{s c}\right)^{2}+\left(\delta_{p}\right)^{2}+\left(\delta_{s t}\right)^{2}+\left(\delta_{n}\right)^{2},
$$

where $\delta_{s c}$ is the intrinsic resolution of the crystal, $\delta_{p}$ is the transfer resolution, $\delta_{s t}$ is the statistical contribution of PMT and $\delta_{n}$ is the dark noise contribution. The intrinsic resolution of a crystal is usually mainly associated with the non-proportional response of the scintillator [8-10]. However, an experi- 
ments show connection of intrinsic resolution with many other phenomena such as inhomogeneity of the scintillator causing local variations in the light output. The statistical uncertainty of the signal from the PMT, can be described as:

$$
\delta_{s t}=2.35 \cdot 1 / N^{1 / 2} \cdot(1+\varepsilon)^{1 / 2},
$$

where $N$ is the number of photoelectrons and $\varepsilon$ is the variance of the electron multiplier gain, which is typically 0.1-0.2 for modern PMTs [8-10]. The PMT contribution can be determined experimentally based on the measured number of photoelectrons and it depends on the light output of the crystal being studied, the quantum efficiency of the photocathode and the efficiency of photoelectron collection.

Last years studies [11-13] of the intrinsic energy resolution nature allows to assume the complex structure of this phenomena and necessity to keep this issue in mind for more precise estimation of the energy resolution value. Kinetics and efficiency of emission and registration of scintillation photons depend on the concentration of excitations in the track region [13]. Some terms in intrinsic energy resolution are connected with fluctuations of the efficiency of photon emission in regions with different concentration of excitations. The fluctuations in track structure produce important additional term in intrinsic energy resolution of scintillators.

These additional terms in energy resolution can be partially compensated if the technique of weighting signals from different time windows is applied [11, 12]. Such assumptions claim for more advanced approach to the energy resolution measurements of that allows to receive better value of the resolution.

In the frame of this study we try check new approach to scintillation photon statistics based on separation of their groups and selection of the part of data that corresponds to the better energy resolution data. Other words, we would like to find only the part of statistical data and cut off the part of the data deteriorated the total statistics.

\section{Experimental}

\subsection{Sample selection}

For the evaluation of processing method $\mathrm{Nal}(\mathrm{Tl})$ scintillation detectors were chosen as the most typical commercially available scintillator. It has to be noted that the spread of experimental data is very wide [14] - from 5.5 to more than $9 \%$ for ${ }^{137}$ Cs source. Such spread allows to assume that many factors contribute to the finally measured energy resolution.

$1 \times 1$ (dia - $25 \mathrm{~mm}$, length $-25 \mathrm{~mm}$ ) $\mathrm{Nal}(\mathrm{Tl})$ detector with $8.6 \%$ resolution was selected as medium or even lower quality. It has to leave a room for investigation of different aspects important for the energy resolution. Each measurement was based on 42000 counts statistics.

\subsection{Spectrum processing method}

The core of the method is layer-by-layer separation of the measured photons database. Previously [12] the separation was completed by decay time curve splitting. It this work the energy spectrum was processed layer-by-layer to reduce the influence of random factors. This set of data was used for later clustering method apply.

The accumulated data were divided into $n S$ layers with a data volume $N s i$ in the $i$-th layer, i.e. $N s i=N / n S$.

A spectrum $S i$ was constructed from the data Nsi using the principle of superposition

$$
N=\sum_{i} N s i, \quad S=\sum_{i} S i
$$

The spectrum $S i$ was divided by energy into $n Z$ sections with a length de in the studied energy range $d E$, i.e. $n Z=d E / d e$. For each $j$-th part of the $n Z j$ spectrum $S i$, a vector model $v_{i j}=\left[p_{1} \ldots p_{n v}\right]$ of size $n v$, was built. $v_{i j}$ - a vector model of the $j$-th part of the $i$-th layer/group of the spectrum, $p_{1} \ldots p_{n v}$ - the parameters of the vector model. Thus, the vector model of the $i$-th layer of the spectrum is a rectangular matrix of size $n Z \times n v$, where $n Z$ is the number of divisions of a single data layer/group, the size of a vector model of the division.

\subsection{Clusterization method}

Last years statistical methods of data analysis were developed for different applications and demonstrated a high efficiency. It is important to note that these methods allows separate uniform from the first glance large data base for several groups and analyze each group separately. This approach allows to extract the most efficient part of the database and finally receive better quality of result analysis [15-22]. For this study was selected vector-matrix model of data representation [22] in particular. 


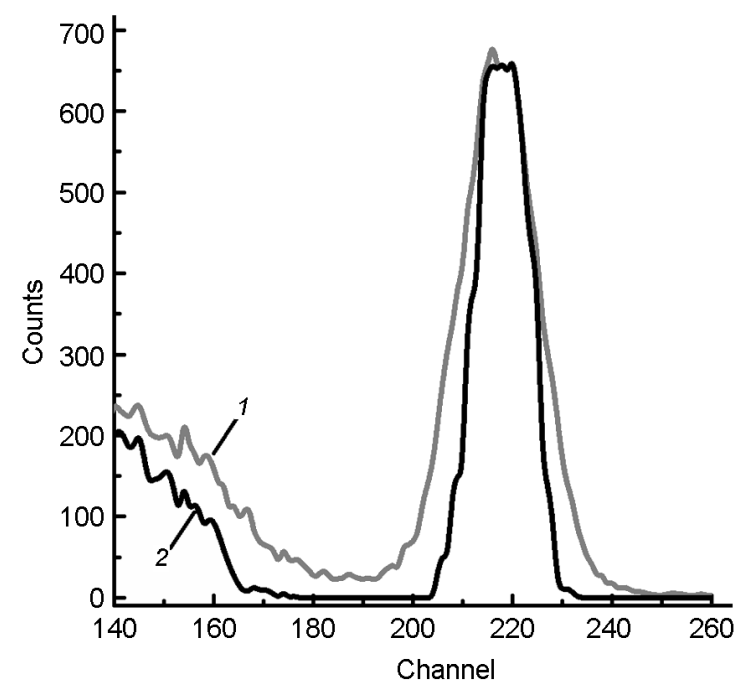

Fig. 1. Initial (curve 1) and processed (curve 2) pulse height spectra acquired with a ${ }^{137} \mathrm{Cs}$ source of a $\mathrm{Nal}(\mathrm{TI})$ crystal.

The method of implementing the matrix data model in hierarchical structures is considered.

A set of rules for structuring information for describing information using a matrix data model is considered. The functionality of the matrix data model is described by mathematical methods of manipulating data based on the operations of the algebra of multidimensional matrices.

Matrix of vector data layer models $N s i$ interpreted as variable observations $n Z$.

The cluster-data hierarchical clustering method was used to form a hierarchical tree of binary clusters. Cluster tree formation is based on combining the two underlying nodes into one overlying node, and so on. The criterion for joining nodes into a cluster is the ratio of the distances between pairs of objects or clusters.

\section{Results and discusson}

In the frame of the vector model (we used in this work) of $i$ layer we use in this study all data ( Nsi) were divided for two clusters $k$, where $k=1$ or 2 .

$$
N s i=N s i_{1}+N s i_{2}
$$

It has to be noted that a priory there are no criteria for amount of clusters. Moreover, separation for three cluster did not change significantly final results.

The square of the Euclidean distance was used as the distance of the cluster points to its centroid.

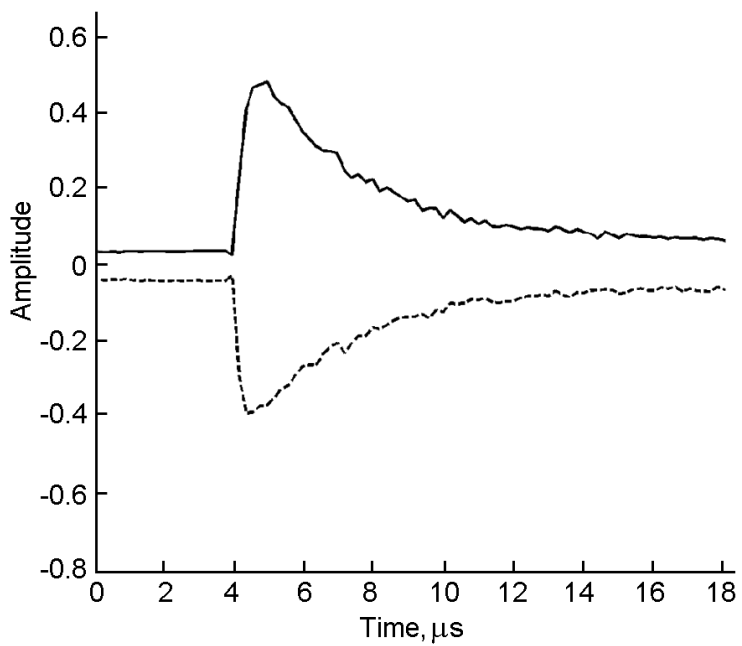

Fig. 2. Deviations of the centroids.

All $n S$ layers were sequentially processed.

From (1) following:

$$
N=\sum_{i} N s i_{1}+\sum_{i} N s i_{2}
$$

For each sum $\sum_{i} N s i_{1}$ and $\sum_{i} N s i_{2}$ the spectra $S 1$ and $S 2$ were estimated, correspondently.

Fig. 1 presents initial (curve 1) and processed (curve 2) pulse height spectra acquired with a ${ }^{137} \mathrm{Cs}$ source of a $\mathrm{Nal}(\mathrm{TI})$ crystal. Even without physically backgrounded cluster separation the best value - $5.66 \%$ - significantly (34\%) better than value for the all photons counting. It means that statistics based on the whole phonons counting do not allows receive an optimal energy resolution.

When tuning the spectrum processing algorithm, the data value, layer size and the length of the segment de were chosen voluntary. At the moment we have no strict physical criteria for selection, but the difference for various option is visible. It means that statistics for some specific photons selection demonstrate better energy resolution value.

The following data was used to analyze the results of spectrum processing:

- on the $S 1$ spectrum data from 218 to 222 channel was taken along the "Channels" axis;

- on spectrum $S 2$ data from 185 to 218 channel and data from 222 to 250 channel were taken along the "Channels' axis.

For each of the three selected data groups, the average pulses were found (centroids, respectively, C1, C21, C22). As the 
distances of the data points, the output pulses of the DM, to the centroid, while minimizing them, the square of the Euclidean distance was used.

Fig. 2 shows the deviations of the C21 centroids (solid line) and the C22 centroids (dashed line) from $\mathrm{C} 1$. The figure shows that the output pulses of the scintillation detector at the absorption peak maximum, up to $7.5 \%$ of the amplitude, differ in shape at a distance of up to 2 microseconds from the front. Over the next 4 microseconds, these differences are reduced by 2 times and over the next 4 microseconds they stabilize at more than four times less than the initial one. It means that decay parameters have to be keep in mind at clusterisation at the future development of more advanced model.

\section{Conclusions}

In conclusion we have to note that proposed advance statistic method allows to get better value of energy resolution comparing to conventional approach. It means that counting all photons add the energy resolution some harmful artefacts. Decrease of $34 \%$ resolution data demonstrate significance of such contribution.

This study as well as previous analysis for decay curves [12] does not allows to fix fundamental reason for this phenomenon but demonstrate efficiency of new statistical approaches to scintillation photons counting and selection.

Statistical methods for processing an array of scintillation photon data show the need for their separation into separate groups and further work with each of them. We cannot say that the chosen method of selection is optimal, but it is enough to create a method of improved energy resolution analysis.

\section{References}

1. R.Hofstadter, Phys. Rev., 74, 100 (1948).
2. P.Dorenbos, J.T.M.De Haas, C.W.E.Van Eijk, IEEE Trans. Nucl. Sci., 42, 2190 (1995).

3. P.Lecoq, A.Gektin, M.Korzhik, Inorganic Scintillators for Detector Systems. Physical Principles and Crystal Engineering, Handbook, 2nd Edition, Springer, New York (2017),

4. M.Nikl, A.Yoshikawa, Adv. Opt. Mater., 3, 463 (2015). DOI: 10.1002/adom.201400571

5. S.E.Derenzo, M.S.Boswell, E.BourretCourchesne et al., IEEE Trans. Nucl. Sci., 55, 1458 (2008).

6. K.Yang, P.Menge, J.Appl.Phys., 118, 213106 (2015).

7. V.Khodyuk, S.A.Messina, T.J.Hayden et al., J.Appl. Physics, 118, 084901 (2015).

8. M.Moszynski, Nucl.Instr.Meth.Phys.Res.A, A505, 101 (2003).

9. M.Moszynski, J.Zalipska, M.Balcerzyk et al., Nucl. Instr. Meth. Phys. Res.A, A484, 259 (2002).

10. M.Moszynski, Proc. SPIE, 5922 (2005).

11. A.V.Gektin, A.N.Vasil'ev, Functional Materials, 24, 621(2017).

12. S.Gridin, D.R.Onken, R.T.Williams et al., $J$. Appl. Phys., 124, 154504 (2018).

13. A.Gektin, A.Vasil'ev, Rad.Meas., 122, 108 (2019).

14. A.Gektin, A.Vasil'ev, Abstr. Int. Conf. Luminescent Detectors and Transformers of Ionizing Radiation (LUMDETR'2018), Prague, Czech Republic (2018), p.221.

15. K.A.Parsaye, J. Data Warehousing, 1 (1998).

16. J.Lee, S.Kim, G.Lebanon et al., J. Machine Learn. Res., 17, 1 (2016).

17. M.S.Aldenderfer, R.K.Blashfield, Cluster Analysis, Sage Publications, Los Angeles (1984). DOI: $10.4135 / 9781412983648$

18. L.Rokach, O.Maimon, Clustering Methods, Data Mining and Knowledge Discovery Handbook, Springer, US (2005),

19. J.Abonyi, B.Feil, Cluster Analysis for Data Mining and System Identification, Boston, MA: Birkhauser, Basel (2007).

20. B.S.Everitt, S.Landau, M.Leese, Cluster Analysis, 4th ed., Arnold, London (2001).

21. K.Jajuga, A.Sokolowski, H.Bock, Classification, Clustering and Data Analysis, Springer New York, (2002). DOI: 10.1007/978-3-642$56181-8$

22. V.P.Sergeev, Bull. Comput. Inform. Technol., 6, 3 (2010). 